\author{
Military Technical College \\ Kobry El-Kobbah, \\ Cairo, Egypt.
}

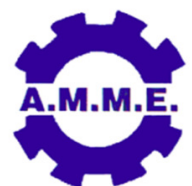

$16^{\text {th }}$ International Conference
on Applied Mechanics and
Mechanical Engineering.

\title{
DYNAMIC MODEL AND ANALYSIS OF A HEXAPOD ROBOT
}

\author{
W. Eldreny ${ }^{*}$, G. Elnashar ${ }^{*}$, M. Hegazi ${ }^{*}$ and A. Badawy ${ }^{*}, 1$
}

\begin{abstract}
This paper presents a dynamic model of a six-legged robot. The direct and inverse kinematic analyses for each leg are considered in order to develop an overall kinematic model of the robot. Feet forces distributions of the hexapod are calculated in order to solve for the dynamic model of the hexapod. Lagrange-Euler formulation is then used to determine the joint torques required for each leg of the hexapod. However, in order to have a better understanding of walking, dynamic stability, energy efficiency, and on-line control, kinematic and dynamic models based on a realistic walking robot design are necessary. Here, an attempt to carry out kinematics, dynamics and optimal feet force distributions of a realistic six-legged robot.
\end{abstract}

\section{KEY WORDS}

Robot dynamics; Legged robot; Path planning; Lagrange-Euler dynamics; Inverse kinematics. 


\section{INTRODUCTION}

Recently, many studies have been carried out on multi-legged walking robots because walking robots offer better mobility. Multi-legged robot has the advantage over the wheeled robot as it used the isolated point to support the robot body not the continuous path that is needed by wheeled robot. All of the advantages make the multi-legged robot become significant and vigorous area of research in the field of mobile robots.

Design of the legged-robot is a complicated problem in both applied mechanics and robotics. It needs solving many problems like kinematics, gait planning, trajectory generation, dynamics, control etc. In order to develop efficient control algorithm of robots, it is important to have good models describing the kinematic and dynamic behaviors of the complex multi-legged robotic mechanism.

To control the motion of the robot, the motion controller calculates the resultant control force-couple system that should be applied to the robot's body by its supporting legs. Therefore, one of the important issues of a legged robot's active force control is the successful distribution of its body force to the feet. For a statically stable multi-legged robot, at least three legs should be on the ground at any instant. If a three-dimensional reaction force vector is considered on each ground leg, the foot force distribution problem becomes indeterminate during the walking because of the closed chain system. Multiple solutions might exist, which can satisfy the forcemoment balance criteria [1-5]. The trajectory in multidimensional space describing motion of a hexapod leg is presented in [6].

Although the above attempt could find the optimal values of feet forces of the multilegged robot, they may not be suitable for real-time implementations because the used optimization techniques are iterative in nature. Moreover, due to inherent complexity of a realistic walking robot, it is not an easy task to include inertial terms in the modeling. The most of the studies on walking dynamics are conducted with a simplified model of legs and body.

\section{KINEMATIC MODEL OF HEXAPOD ROBOT}

The coordinate frames of reference for the robot legs following the DenavitHartenberg notation are assigned as shown in Fig. 1[7]. Let the reference frame of the hexapod robot at the center of mass of its body $G_{00}$ so that $Z_{00}$ is vertical and $X_{00}$ refer to the direction of walking forward. Let the reference axis of each leg at $\mathrm{G}_{0 \text { n }}$ where $n$ is the leg number (1 to 6 ) as shown in Fig. 2. The kinematic equation of the hexapod leg has the form of:

$$
T_{n 4}^{00}=\left[\begin{array}{cccc}
c_{23} c_{1} & -s_{23} c_{1} & s_{1} & d x_{n}+c_{1}\left(L_{2} c_{23}+L_{1} c_{2}\right) \\
c_{23} s_{1} & -s_{23} s_{1} & -c_{1} & d y_{n}+s_{1}\left(L_{2} c_{23}+L_{1} c_{2}\right) \\
s_{23} & c_{23} & 0 & d z_{n}+L_{2} s_{23}+L_{2} s_{2} \\
0 & 0 & 0 & 1
\end{array}\right]
$$




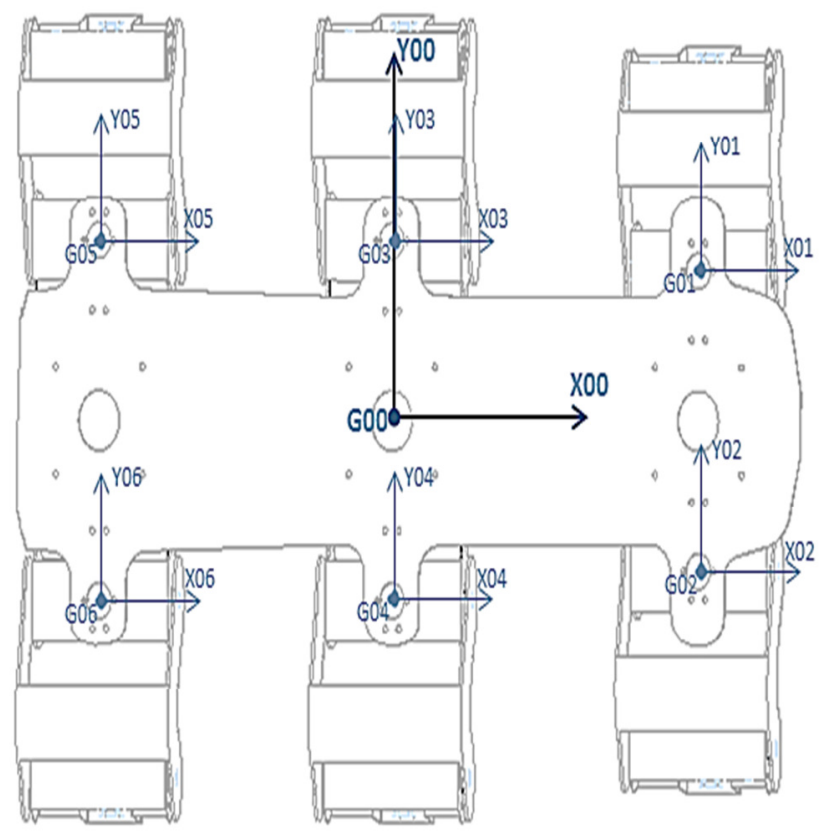

Fig. 1. Hexapod legs reference frames relative to body reference frame

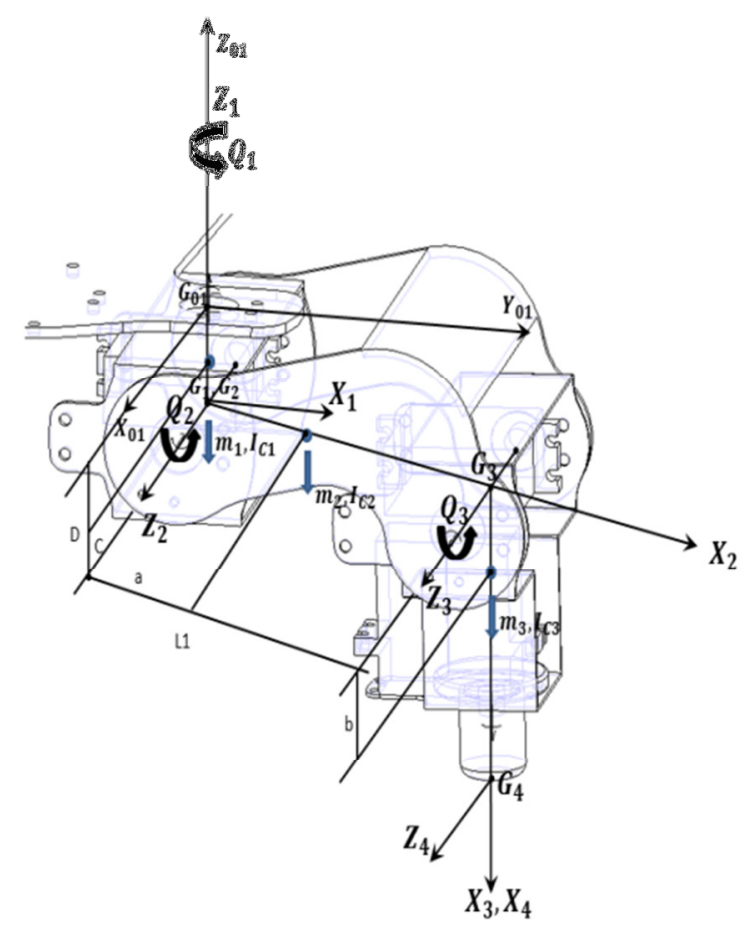

Fig. 2. D-H representation of the hexapod leg.

Given a desired position $(x, y, z)$ of the foot tip of the hexapod leg, the joint variables are then calculated. Inverse kinematic problem of 3 DoF leg is solved algebraically using Paul method $[7,8]$ as following:

$$
P_{X} c_{1}+P_{Y} s_{1}=L_{2} c_{23}+L_{1} c_{2}
$$




$$
\begin{gathered}
P_{Y} c_{1}-P_{X} s_{1}=0 \\
P_{Z}=-L_{2} s_{23}-L_{2} s_{2}
\end{gathered}
$$

Let

$$
K=\frac{P_{Z}^{2}+P_{X}^{2}+P_{Y}^{2}-L_{1}^{2}-L_{2}^{2}}{2 L_{1}}
$$

Then

$$
\begin{gathered}
\theta_{1}=\tan ^{-1}\left(\frac{P_{Y}}{P_{X}}\right) \\
\theta_{3}=\tan ^{-1}\left(\frac{ \pm \sqrt{\left(L_{2}^{2}-\mathrm{K}^{2}\right)}}{K}\right) \\
\theta_{23}=\tan ^{-1}\left(\frac{\left(L_{1} c_{3}+L_{2}\right) P_{Z}+\left(P_{X} c_{1}+P_{Y} s_{1}\right) L_{1} s_{3}}{\left(L_{1} c_{3}+L_{2}\right)\left(P_{X} c_{1}+P_{Y} s_{1}\right)-P_{Z} L_{1} s_{3}}\right) \\
\theta_{2}=\theta_{23}-\theta_{3}
\end{gathered}
$$

where $c_{i}=\cos \left(\theta_{i}, c_{i j}=\cos \left(\theta_{i}+\theta_{j}\right), s_{i}=\sin \left(\theta_{i}\right), s_{i j}=\sin \left(\theta_{i}+\theta_{j}\right)\right.$.

Jacobian matrix $\mathbf{J}^{0}$ of the hexapod leg is a time varying linear transformation that relates leg's joint rates to Cartesian velocity of the foot tip of hexapod leg. By inverting the Jacobian matrix joint rates are obtained via knowing the Cartesian velocity of the tip of hexapod leg as:

$$
\mathbf{J}^{0}=\left[\begin{array}{ccc}
-s_{1}\left[L_{2} c_{23}+L_{1} c_{2}\right] & -c_{1}\left[L_{2} s_{23}+L_{1} s_{2}\right] & -L_{2} s_{23} c_{1} \\
c_{1}\left[L_{2} c_{23}+L_{1} c_{2}\right] & -s_{1}\left[L_{2} s_{23}+L_{1} s_{2}\right] & -L_{2} s_{23} s_{1} \\
0 & L_{2} c_{23}+L_{1} c_{2} & L_{2} c_{23}
\end{array}\right]
$$

\section{PATH GENERATION AND MOTION CONTROL}

The trajectory in multidimensional space, which describes the desired motion of a hexapod leg, is an important factor in studying the dynamic model of the hexapod robot during robot walking [6]. Speed is also an extremely significant factor for robot locomotion. It depends on the step width $(W)$, the cycle time $(T)$, the duty factor $\beta$, and the number of legs. The flying phase is configuration where the leg is in the air in order to make the next step. Whereas the support phase is the configuration, where the leg touches the ground and moves the robot forward within. The ratio of the duration of the support phase to the total step time is called the duty factor. Robot velocity is defined accordingly as:

$$
v_{n}=\frac{W}{t_{s}}=\frac{W}{t_{f}}\left(\frac{1-\beta}{\beta}\right)
$$


Figure 3 shows a wave gait with $\beta=1 / 2$, which is the most common for hexapod robots since it produces the smallest duty factor for hexapods and thus results in the fastest walking speed while keeping static stability. This gait is called a tripod gait since the three legs $1,4,5$ or 2, 3, 6, support a robot [9].
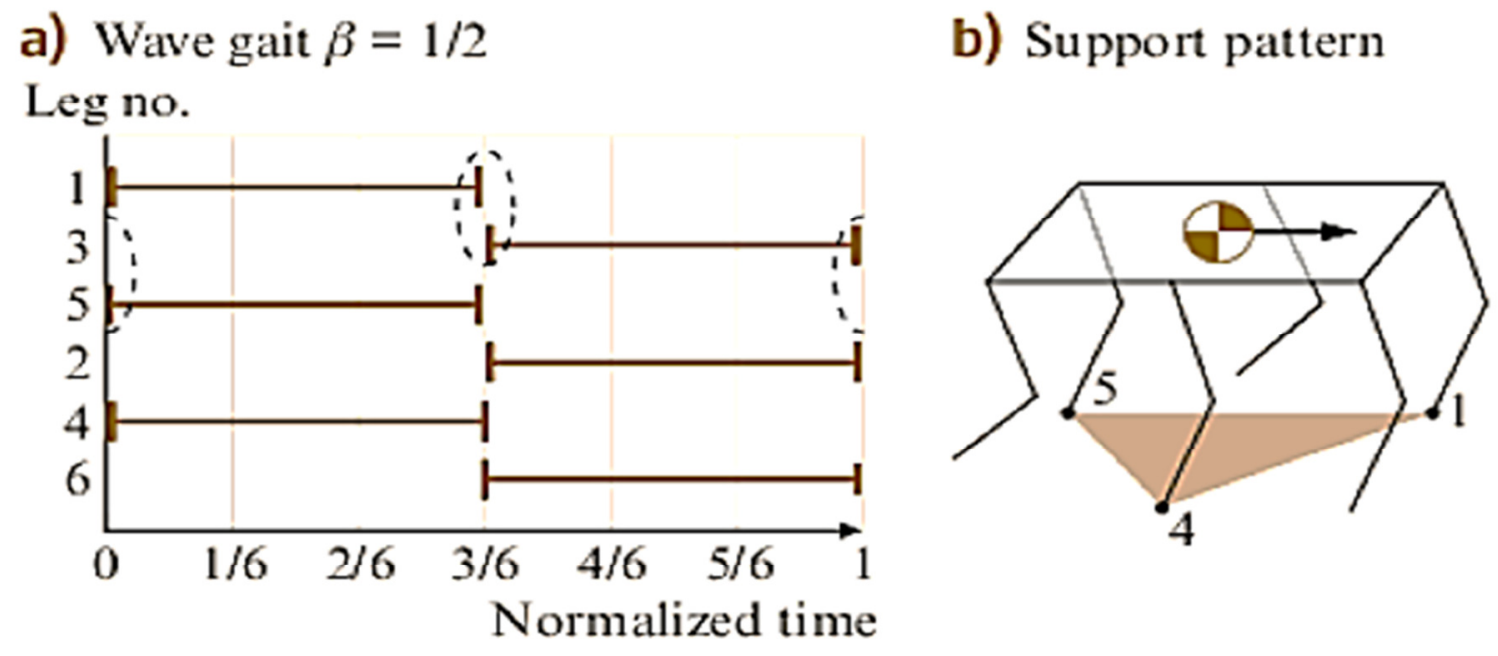

Fig. 3. Tripod gait of a hexapod robot.

Step cycle consist of two phases: $[A]$ and $[B]$. Legs number 1,4 and 5 will be in phase $[A]$, while legs number 2, 3 and 6 will be in phase $[B]$. Moreover, after the time equal $[T]$ the phases will exchange. By knowing the step parameters, the path of the step of every hexapod leg in the Cartesian coordinate relative to body reference frame is determined as shown in Fig. 4 using the following control parameters:

$\left[\mathrm{P}_{\mathrm{i}}\right]$ is the start point of the path of the leg tip.

$\left[\mathrm{P}_{\mathrm{f}}\right]$ is the end of the path of the leg tip.

$\left[P_{m}\right]$ is the via point at the middle of the path of the leg tip.

$[T]$ is the step duration time.

Using inverse kinematic model of the hexapod leg it is possible to solve for these three points and then obtain the three configurations of the leg joints. Boundary conditions for the two phases are shown in Fig. 4.

To make the hexapod leg move smoothly, every joint angle starts and ends at rest with zero acceleration. A higher order polynomial of the $7^{\text {th }}$ degree is suitable to describe the path of every joint angle. Results for the joints angles, velocities, and accelerations relative to the time during step path are shown in Fig. 5.

Figure 5, also, gives the required span of each joint. For instance, joint (1) ranges from $75^{\circ}$ to $105^{\circ}$ so it is now able to install some suitable motor covering this range unlike joint (2) which rotate from an angle $-10^{\circ}$ to $15^{\circ}$. Moreover, maximum allowable angular speed of each actuator is also determined. 


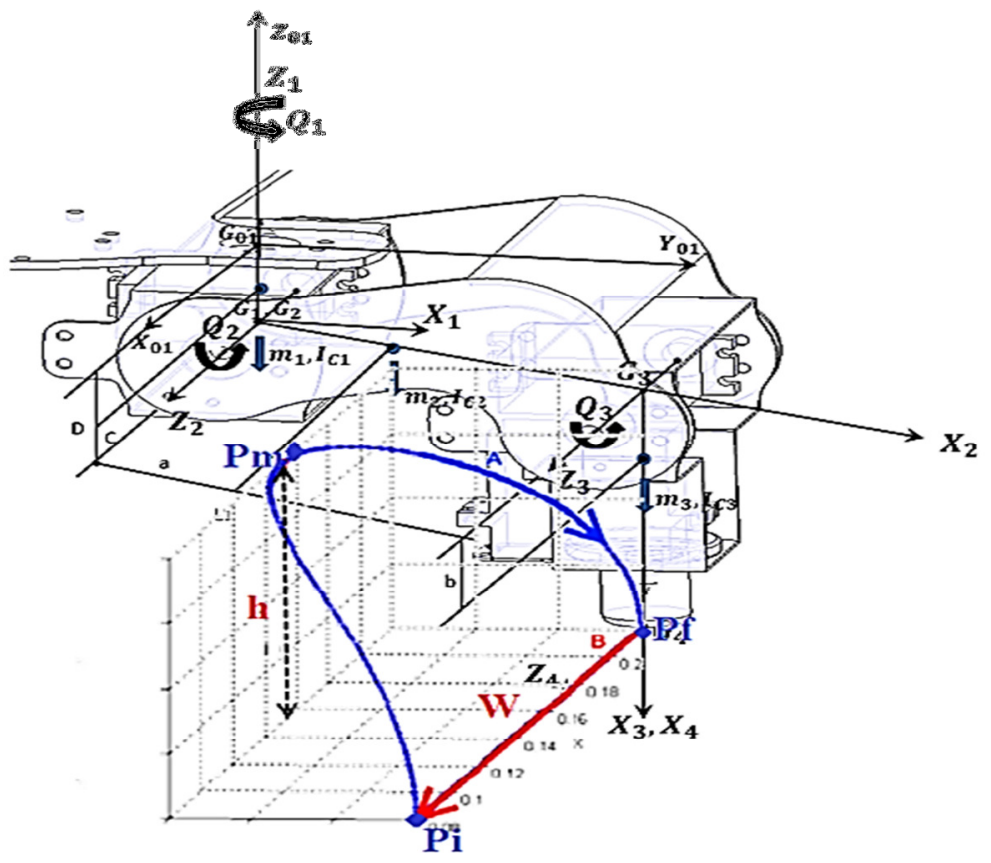

Fig. 4. The path of leg tip in the Cartesian space.
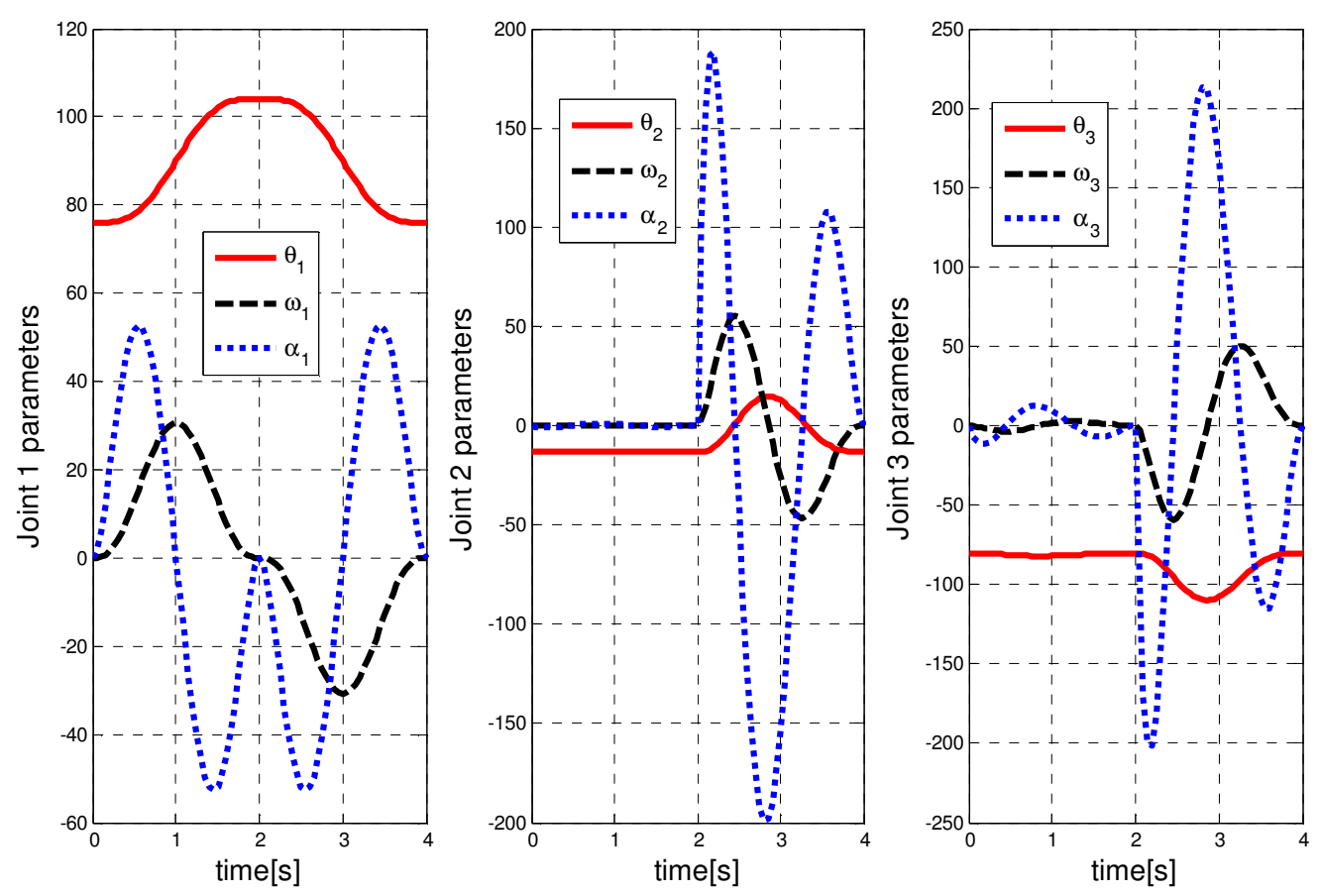

Fig. 5. Joint angles, velocities, and accelerations during step cycle.

\section{HEXAPOD DYNAMIC EQUATIONS}

The objective of the hexapod dynamic equations is to determine all joint torques' variations over a hexapod robot step cycle. Lagrange dynamics formulation is expressed in the vector-matrix form as follows [6]: 


$$
[Q]_{i}=[M(\theta)]_{i}[\ddot{\theta}]_{i}+[B(\theta)]_{i}[\ddot{\theta} \dot{\theta}]_{i}+[C(\theta)]_{i}\left[\dot{\theta}^{2}\right]_{i}+[G(\theta)]_{i}+[J]_{i}^{T}[f]_{i}
$$

where :

$\begin{array}{ll}{[\mathrm{M}(\theta)]_{\mathrm{i}}} & \text { is the } 3 \times 3 \text { mass matrix of } \mathrm{i}^{\text {th }} \text { leg. } \\ {[\mathrm{B}(\theta)]_{\mathrm{i}}} & \text { is the } 3 \times 3 \text { matrix of Coriolis coefficient of } \mathrm{i}^{\text {th }} \text { leg } \\ {[\mathrm{C}(\theta)]_{\mathrm{i}}} & \text { is the } 3 \times 3 \text { matrix of centrifugal coefficient of } \mathrm{i}^{\text {th }} \text { leg } \\ {[\mathrm{J}]_{\mathrm{i}}^{\mathrm{T}}} & \text { is the } 3 \times 3 \text { Jacobin matrix of } \mathrm{i}^{\text {th }} \text { leg. } \\ {[\mathrm{G}(\theta)]_{\mathrm{i}}} & \text { is the } 3 \times 1 \text { vector of gravity terms of } \mathrm{i}^{\text {th }} \text { leg. } \\ {[\mathrm{Q}]_{\mathrm{i}}} & \text { is the } 3 \times 1 \text { vector of joints torques of } \mathrm{i}^{\text {th }} \text { leg } . \\ {[\mathrm{f}]_{\mathrm{i}}} & \text { is the } 3 \times 1 \text { vector of ground reaction forces of } \mathrm{i}^{\text {th }} \text { leg. }\end{array}$

\section{GROUND REACTION FORCE DISTRIBUTION ON HEXAPOD LEGS}

The following assumptions are made for computing foot-force distributions:

1. No slip occurs at the tip point of all legs throughout the support phase.

2. The contacts of the tip of all feet with ground are modeled as hard point contacts with friction.

3. The effect of inertia of flying legs on trunk body is negligible; transfer legs exert no forces on the trunk body.

During the leg's flying phase, there is no foot-terrain interaction, and the ground reaction forces become equal to zero. However, during the support phase, ground contact exists and system of equations become indeterminate, which has to be solved using an optimization criterion, e.g., optimal foot force distribution $[10,11]$. The ground-reaction force vector on foot [i] at the tip point of the leg is defined as, Fig. 6:

$$
\mathbf{f}_{i i}=\left[\begin{array}{lll}
f_{i_{x}} & f_{i_{y}} & f_{i_{z}}
\end{array}\right]^{T}
$$

The position vector of the tip point of leg number [i] relative to the origin of the reference frame is defined as:

$$
\mathbf{P}_{i}=\left[\begin{array}{lll}
x_{i} & y_{i} & z_{i}
\end{array}\right]^{T}
$$

where $\mathrm{i}=1,2, \ldots, \mathrm{m}$ where $\mathrm{m}$ is the number of ground legs at a particular instant (for example, $m=3$ for tripod gait). The force-couple system that contains the forces and moments that acting on the robot's center of gravity, and represents the robot's payload, including the effect of surface gradient, external forces and inertial effects of the robot's body is then defined as:

$$
\mathbf{W}=\left[\begin{array}{llllll}
F_{x} & F_{y} & F_{z} & M_{x} & M_{y} & M_{z}
\end{array}\right]^{T}
$$

For tripod gate, there exists two phase of motions. Legs number $(i=1,4,5)$ are in phase and legs number $(i=2,3,6)$ are in another phase and these legs change their 


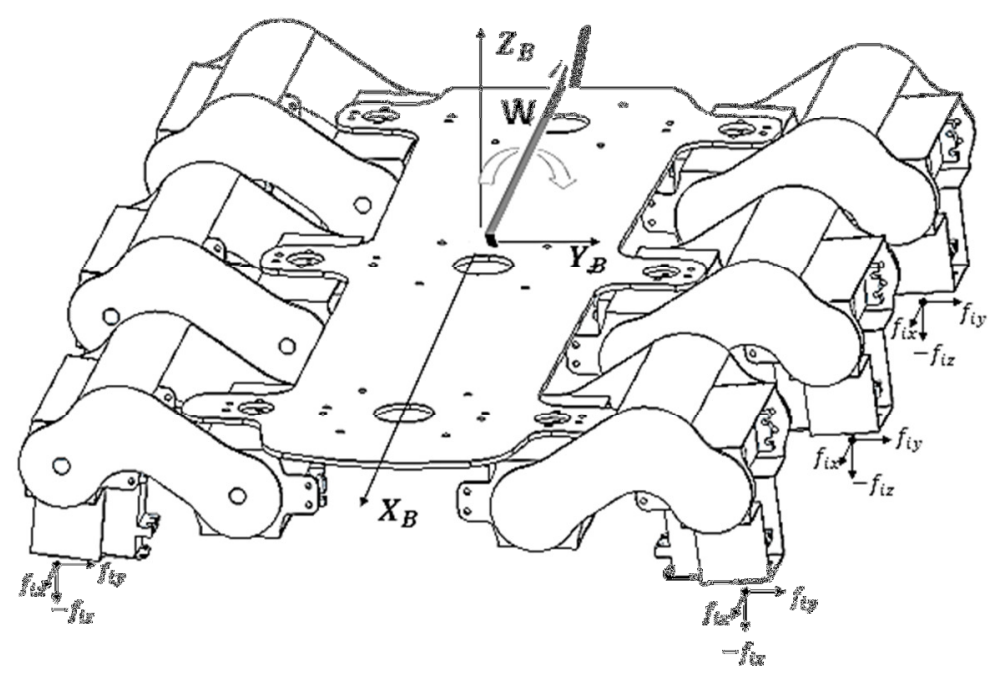

Fig. 6 Reaction force distribution on hexapod legs' tips

phase during the step cycle. Under these conditions and for statically stable system, equilibrium equations are defined as:

$$
\sum_{i} \overrightarrow{\mathbf{f}}_{\mathbf{i}}=-\overrightarrow{\mathbf{F}}, \sum_{\mathbf{i}} \overrightarrow{\mathbf{P}}_{\mathbf{i}} \times \overrightarrow{\mathbf{f}}_{\mathbf{i}}=-\overrightarrow{\mathbf{M}}
$$

This leads to a system of 6 equations in 9 unknowns as:

$$
\left[\begin{array}{ccccccccc}
1 & 0 & 0 & 1 & 0 & 0 & 1 & 0 & 0 \\
0 & 1 & 0 & 0 & 1 & 0 & 0 & 1 & 0 \\
0 & 0 & 1 & 0 & 0 & 1 & 0 & 0 & 1 \\
0 & -z_{1} & y_{1} & 0 & -z_{4} & y_{4} & 0 & -z_{5} & y_{5} \\
z_{1} & 0 & -x_{1} & z_{4} & 0 & -x_{4} & z_{5} & 0 & -x_{5} \\
-y_{1} & x_{1} & 0 & -y_{4} & x_{4} & 0 & -y_{5} & x_{5} & 0
\end{array}\right]\left[\begin{array}{l}
f_{x_{1}} \\
f_{y_{1}} \\
f_{z_{1}} \\
f_{x_{4}} \\
f_{y_{4}} \\
f_{z_{4}} \\
f_{x_{5}} \\
f_{y_{5}} \\
f_{z_{5}}
\end{array}\right]=\left[\begin{array}{c}
-F_{x} \\
-F_{y} \\
-F_{z} \\
-M_{x} \\
-M_{y} \\
-M_{z}
\end{array}\right]
$$

Knowing the feet positions, the feet forces during the hexapod step cycle are computed using the least squared method, which gives the minimum norm solution of the indeterminate equilibrium equations. In other words, the solution minimizes the sum of the squares of components of feet forces [9]. Solution of eq. (14) for a step cyclic time of $4 \mathrm{sec}$. using MATLAB software is presented in Fig. 7.

The total reaction force acts on leg number 4 is greater than the sum of the total reaction force affect legs number 1 and 5 . In addition, the sum of reaction forces affected on the three legs, essentially, equals the total weight of the hexapod robot. Now after calculating the reaction force distribution on hexapod feet, which are in 
support phase, the derivation of the dynamic model of the 3DOF robot leg using Lagrange dynamic formulation in the model in matrix form is presented in the next section.

\section{LAGRANGE DYNAMIC FORMULATION}

The general motion equation of robot leg is expressed through the direct application of the Lagrange dynamic formulation to non-conservative systems [8]. This formulation relies on energy equation and gives the dynamic model equations in a well structure form. Moreover, Lagrange formulation is expressed explicitly in vector matrix form suitable for control analysis and designing a controller in state space as $[6,9]$ :

$$
\frac{d}{d t}\left[\frac{\partial T}{\partial \dot{q}_{i}}\right]-\left[\frac{\partial T}{\partial q_{i}}\right]+\left[\frac{\partial U}{\partial q_{i}}\right]=Q_{i}
$$

where $T$ is the total kinetic energy of the hexapod leg, $U$ is the total potential energy of the hexapod leg, $q_{i}$.is the generalized coordinate of the hexapod leg, $\dot{q}_{1}$.is the generalized velocity of the hexapod leg and $Q_{i}$ is the generalized torque applied to the system at joint $i$.
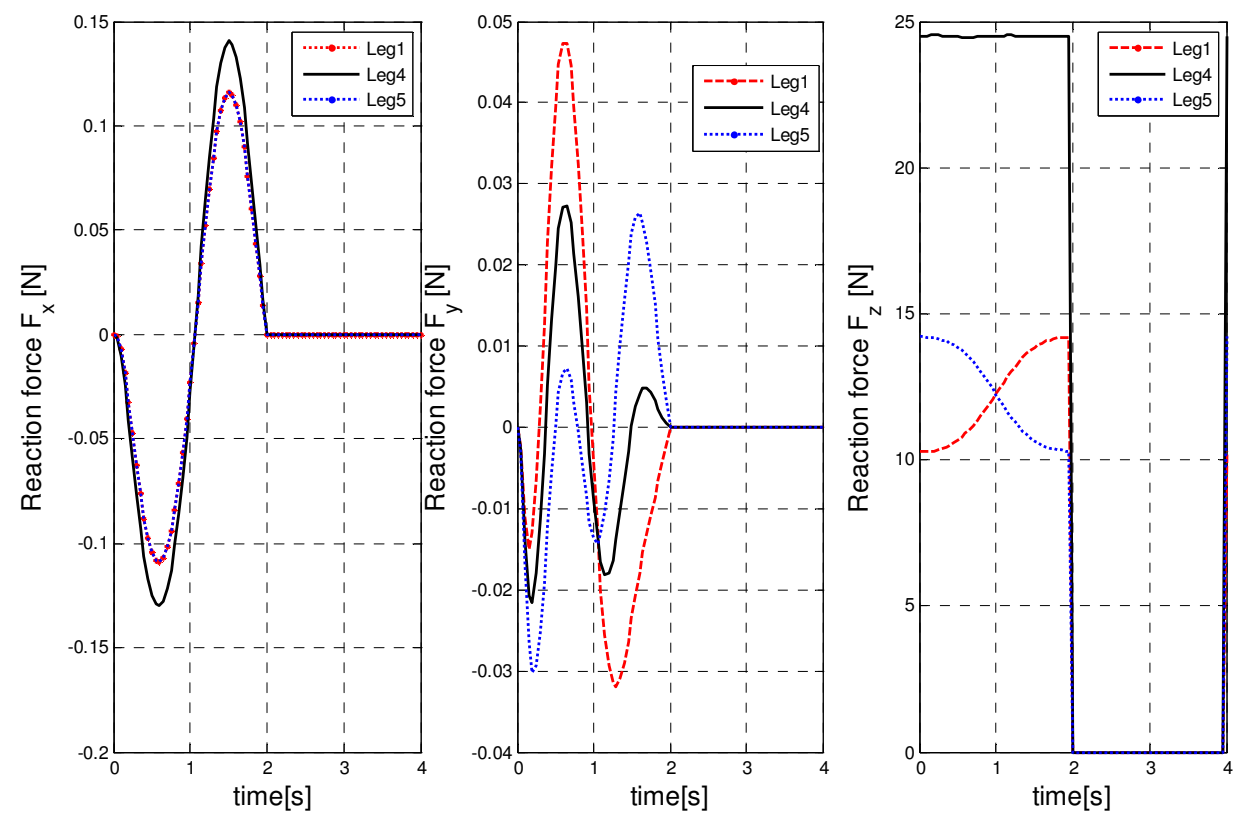

Fig. 7. Reaction forces components for legs number (1, 4, and 5) that in support phase.

Parameters for all links, constitute each leg, are now computed and summed then substituted in eq. (15) as:

$$
\begin{aligned}
& T_{1}=1 / 2 m_{1} v_{C 1}^{T} v_{C 1}+1 / 2 \omega_{1}^{1 T} I_{C 1} \omega_{1}^{1}=1 / 2 I_{z z_{1}} \dot{\theta}_{1}^{2} \\
& U_{1}=-m_{1} g^{0^{T}} r_{C 1}^{0}
\end{aligned}
$$




$$
\begin{aligned}
& T_{2}=1 / 2 I_{y y_{2}} \dot{\theta}_{1}^{2}+1 / 2 I_{z z_{2}} \dot{\theta}_{2}^{2}-I_{y z_{2}} \dot{\theta}_{1} \dot{\theta}_{2}+1 / 2 a^{2} m_{2}\left[c_{2}^{2} \dot{\theta}_{1}^{2}+\dot{\theta}_{2}^{2}\right] \\
& U_{2}=-m_{2} g^{0^{T}} r_{C 2}^{0} \\
& T_{3}=1 / 4 m_{3}\left[L_{1}^{2} \dot{\theta}_{1}^{2}+2 L_{1}^{2} \dot{\theta}_{2}^{2}+b^{2} \dot{\theta}_{1}^{2}+2 b^{2} \dot{\theta}_{2}^{2}+2 b^{2} \dot{\theta}_{3}^{2}+b^{2} \cos \left(2 \theta_{2}+2 \theta_{3}\right) \dot{\theta}_{1}\right. \\
& +4 b^{2} \dot{\theta}_{2} \dot{\theta}_{3}+L_{1}^{2} \cos \left(2 \theta_{2}\right) \dot{\theta}_{1}^{2}+2 L_{1} b c_{3} \dot{\theta}_{1}^{2}+4 L_{1} b c_{3} \dot{\theta}_{2}^{2} \\
& U_{3}=-m_{3} g^{0^{T}} r_{C 3}^{0}
\end{aligned}
$$

After differentiating and then substituting in eq. (15), equation of motion is then expressed as:

$$
\left[\begin{array}{l}
Q_{1} \\
Q_{2} \\
Q_{3}
\end{array}\right]=\left[\begin{array}{lll}
m_{11} & m_{12} & m_{13} \\
m_{12} & m_{22} & m_{23} \\
m_{13} & m_{23} & m_{33}
\end{array}\right]\left[\begin{array}{l}
\ddot{\theta}_{1} \\
\ddot{\theta}_{2} \\
\ddot{\theta}_{3}
\end{array}\right]+\left[\begin{array}{lll}
b_{11} & b_{12} & b_{13} \\
b_{21} & b_{22} & b_{23} \\
b_{31} & b_{32} & b_{33}
\end{array}\right]\left[\begin{array}{l}
\dot{\theta}_{1} \dot{\theta}_{2} \\
\dot{\theta}_{1} \dot{\theta}_{3} \\
\dot{\theta}_{2} \dot{\theta}_{3}
\end{array}\right]+\left[\begin{array}{lll}
c_{11} & c_{12} & c_{13} \\
c_{21} & c_{22} & c_{23} \\
c_{31} & c_{32} & c_{33}
\end{array}\right]\left[\begin{array}{l}
\dot{\theta}_{1}^{2} \\
\dot{\theta}_{2}^{2} \\
\dot{\theta}_{3}^{2}
\end{array}\right]+\left[\begin{array}{l}
G_{1} \\
G_{2} \\
G_{3}
\end{array}\right](16)
$$

All coefficients are determined as functions of links mass, inertia, dimensions, and orientations. Results of path generator are implemented for the robot leg shown in Fig. 4. These results are then introduced in eq. (16) to determine joint torques as functions of time as shown in Fig. 8. Using the forward dynamic model, the Cartesian position, velocity, and acceleration of the hexapod leg tips are calculated and demonstrated Fig. 9.
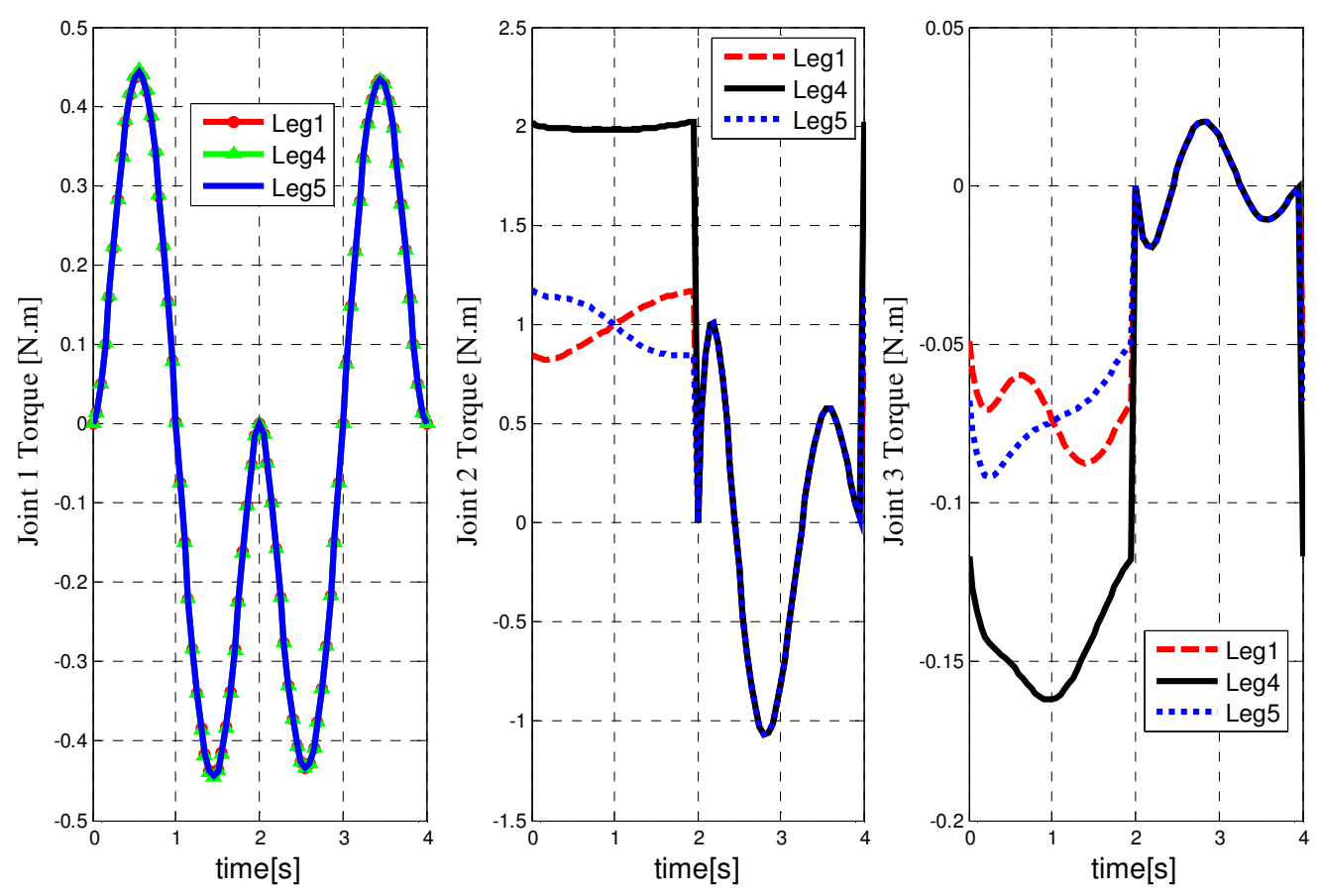

Fig. 8. Torque at the three joints of robot legs including reaction force effect. 

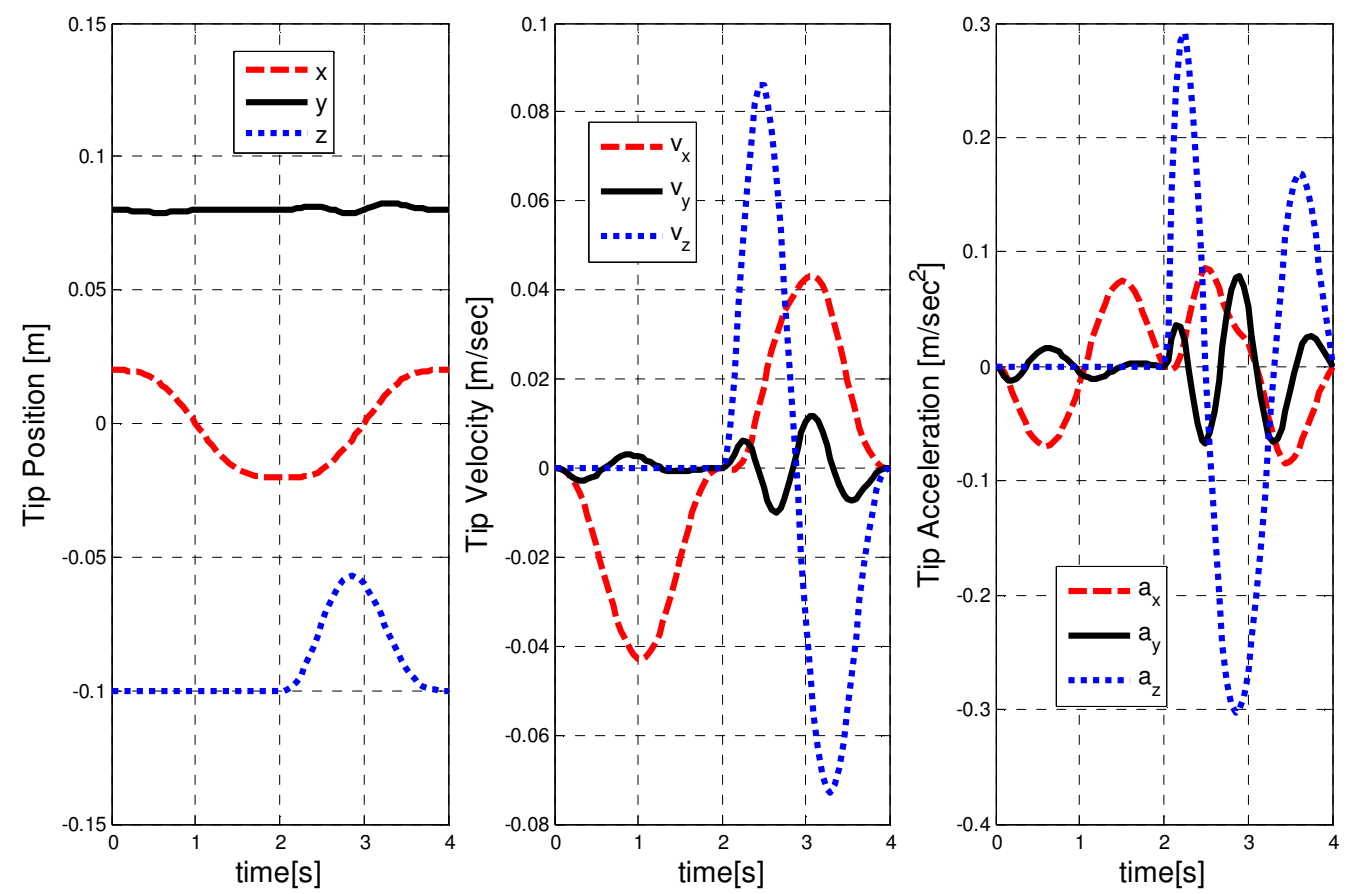

Fig. 9. The tip Cartesian position, velocity, and acceleration of one leg of the hexapod during step cycle.

\section{EFFECT OF STEP PARAMETERS ON THE DYNAMIC PERFORMANCE IN FLYING PHASE}

Now using the dynamic model of the hexapod robot we can study the effect of changing the step parameters as step width, step height, and step angle on joint dynamics during flying phase as shown in Figs. 10, 11, and 12 where the step time is one second.

Figure 10, presents five choices for step width ranging from $10 \mathrm{~mm}$ to $90 \mathrm{~mm}$. Other parameters such as step height and step angle are kept constant. For this simulation, step height is $60 \mathrm{~mm}$ and step angle is zero. Remarkable increase in joint (1) torque as a result of increasing step width, while that for joint (2) is behaving contradictorily. Minor effect on joint (3) torque takes place.

In the second case, both step width and step angle are kept constant. Step width is $50 \mathrm{~mm}$, and step angle remains zero, while Step height is ranging from $10 \mathrm{~mm}$ to 90 $\mathrm{mm}$. Torque of joint (1) is constant regardless changing step height, as this joint motion provides no vertical displacement. On the other hand, other two joints experience considerable change in their torques, Fig. 11.

At last, while considering step angle variation it is noted that whilst the step angle is $90^{\circ}$ joint (1) torque is zero. Deviation away from this angle, in both directions, increases joint (1) torque remarkably. This deviation has minor effect on joint (2) torque, and negligible effect on joint (3) torque, Fig. 12. 

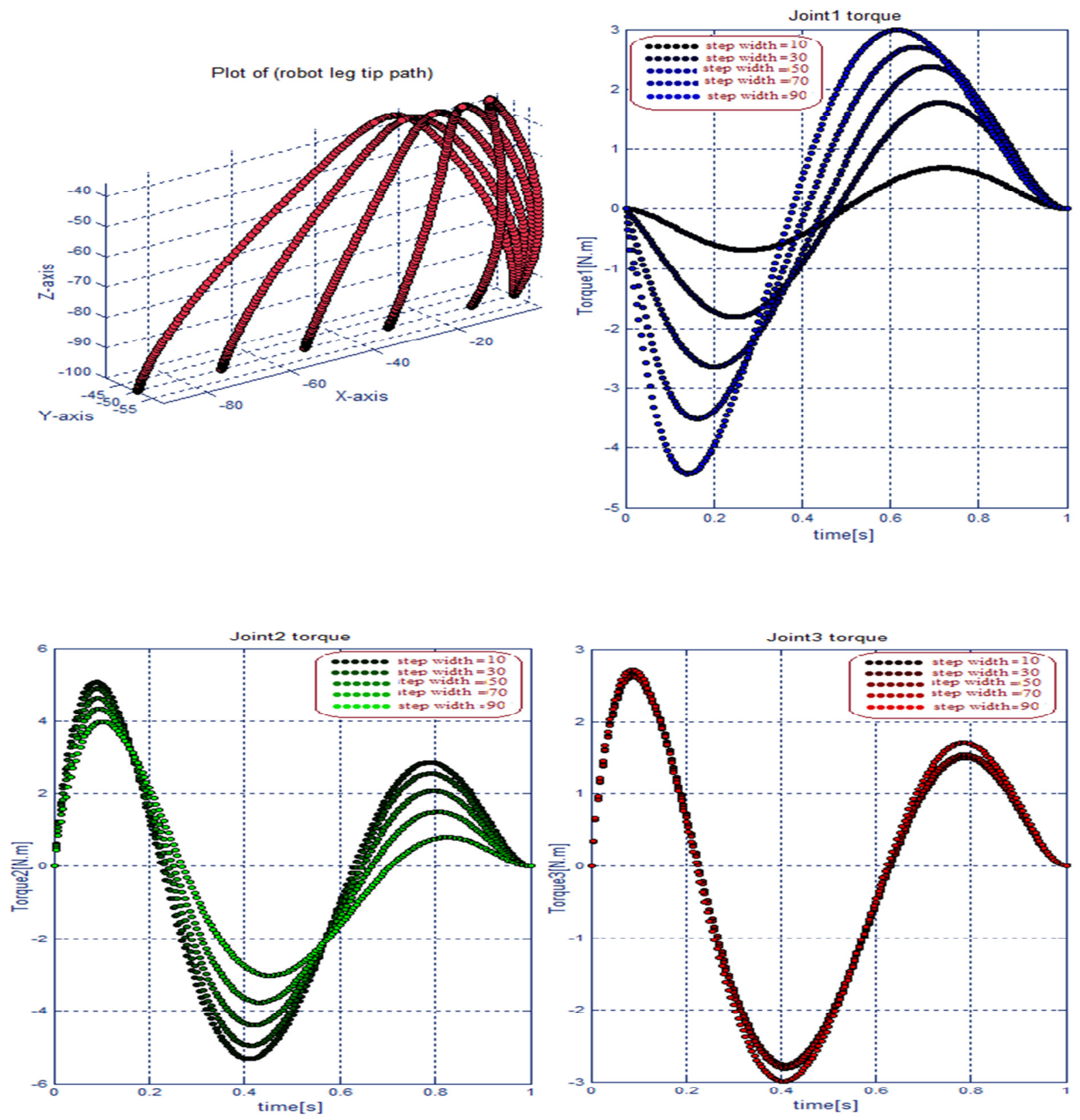

Fig. 10. Effect of hexapod step width on the leg joints' torque in flying phase. 

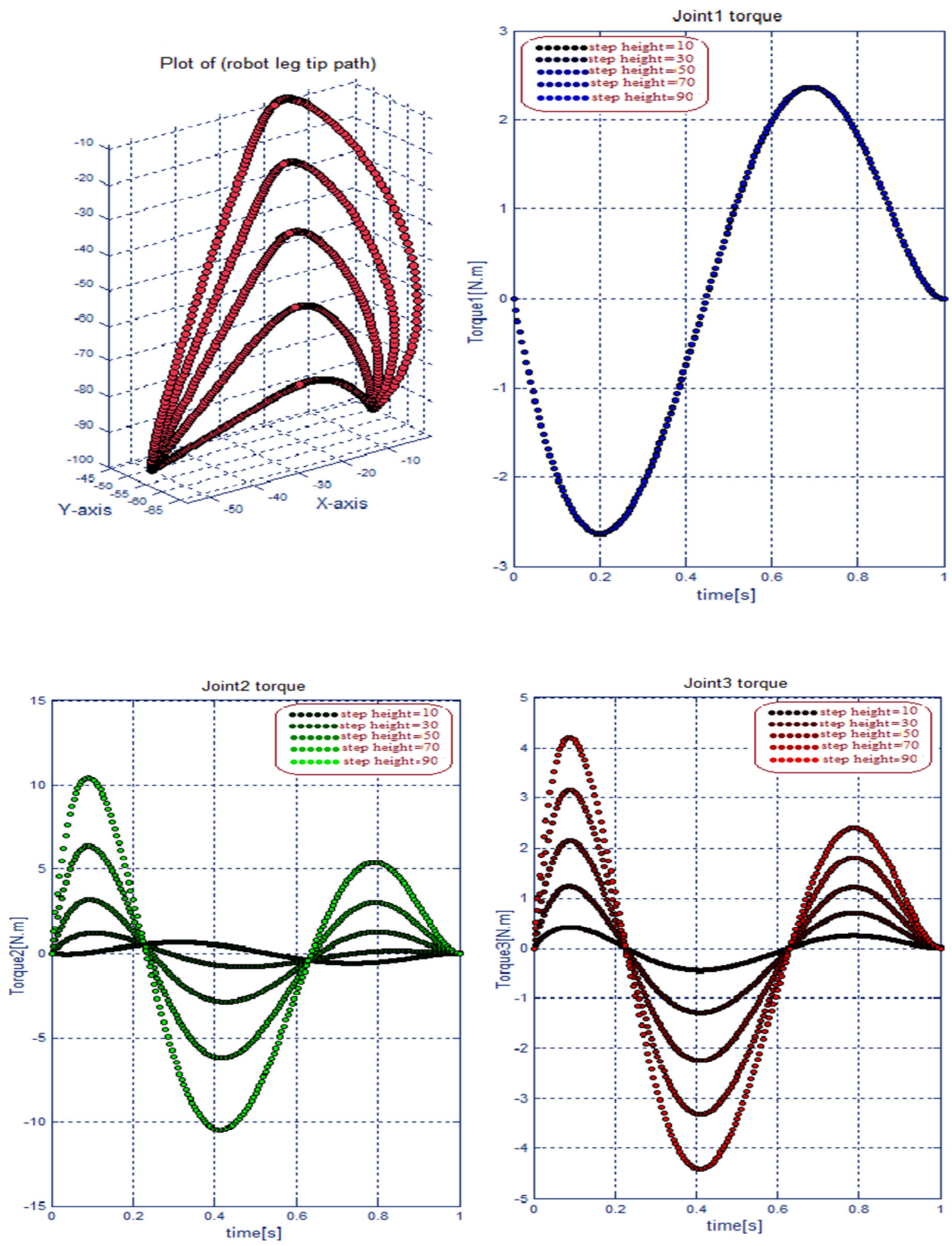

Fig. 11. Effect of hexapod step height on the leg joints' torque in flying phase. 

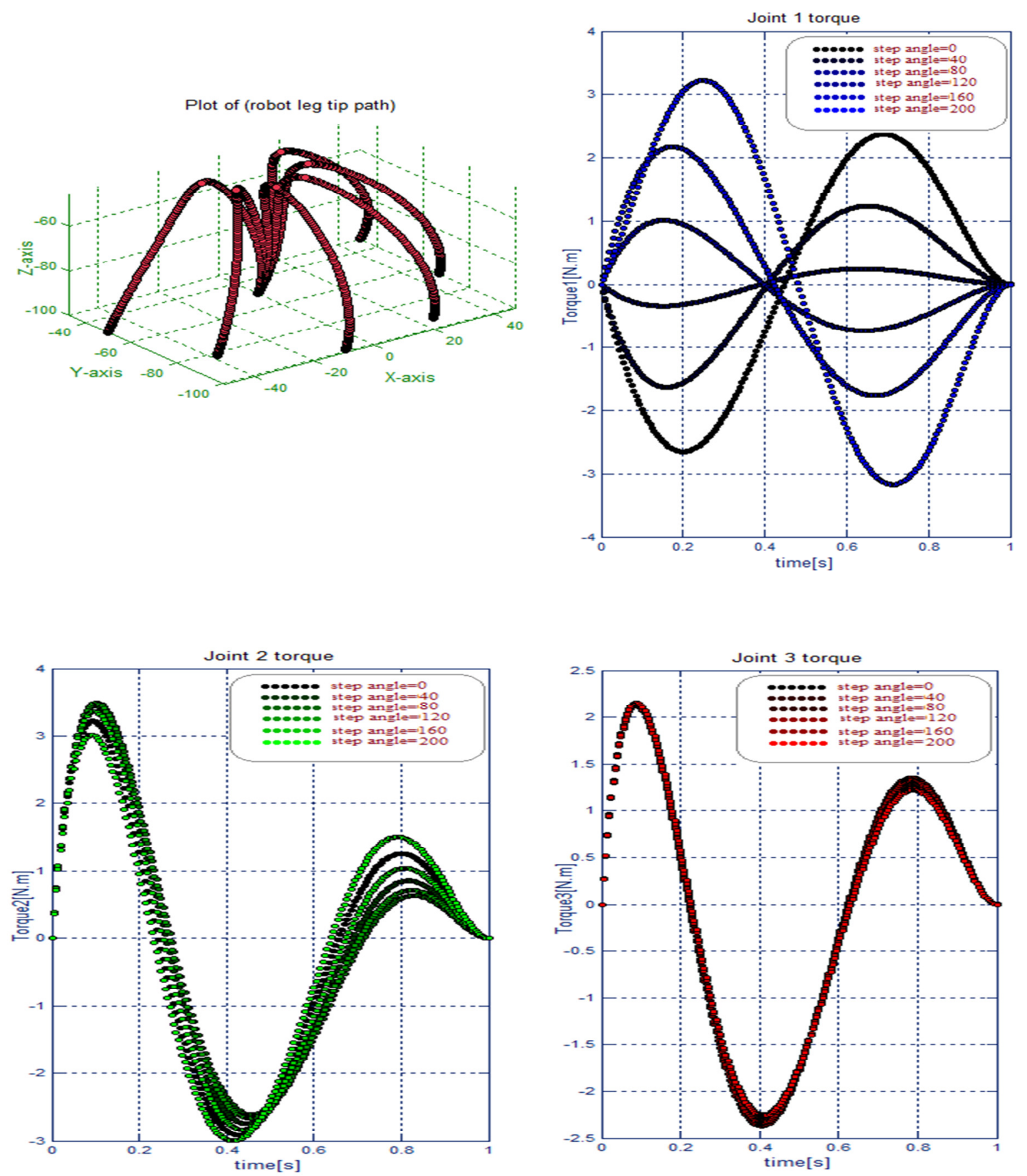

Fig. 12. Effect of hexapod step angle on the leg joints' torque in flying phase.

\section{CONCLUSIONS}

This paper proposed a full dynamic model for a hexapod moving in a straight line. A higher order polynomial of the $7^{\text {th }}$ degree gives a smooth path generation for the model under consideration. Performance of the hexapod is controlled via selecting step parameters: step width, step height, and step angle through which step time and hexapod speed are then calculated. The required span of each joint is 
determined that permit installing some suitable motor covering this range. Moreover, maximum allowable angular speed of each motor is also determined.

An algorithm using the least squared method is used to solve for the indeterminate equilibrium equation. The produced results show that the total reaction force acts on leg number 4 is greater than the sum of the total reaction force affect legs number 1 and 5. This difference is referred to the spatial distribution of these legs relative to the robot body. In addition, the sum of reaction forces affected on the three legs equals the total weight of the hexapod robot all over the cycle that validates the model. The hexapod will accordingly walk in a fashion that affects the joint kinematical parameters that along with the ground force reactions determine joint torques required to perform successful robot maneuver.

Torques for joint number (1), gives similar results for all the three legs for both the support and the flying phases. Whereas for joints number (2) and (3) torques at leg number (4) are noticeably greater than the sum of the torques required for legs number (1) and (5). This difference is referred to the disparity in ground forces apply to each leg. During the flying phase, analogous joints in all legs require the same torques since legs are in fact identical.

By studying of the effect of the step parameters on the joints torques of the robot we can select the optimum step width, step height, and step angle that achieve the minimum joints torques during robot walking.

So the proposed method proved its ability to solve for the dynamic problem of hexapod and is useful in analyzing the motion of the hexapod robot.

\section{REFERENCES}

[1] D. Howard, S.J. Zhang, D.J. Sanger, "Kinematic analysis of a walking machine," Mathematics and Computers in Simulation, vol. 41, pp. 525-538, 1996.

[2] D.M. Gorinevsky, A.Y. Shneider, "Force control in locomotion of legged vehicles over rigid and soft surfaces", The International Journal of Robotics Research, vol. 9(2), pp. 4-23, 1990.

[3] W.Y. Jiang, A.M. Liu, D. Howard, "Foot-force distribution in legged robots," Proc. of 4th Int. Conf. in Climbing and Walking Robots, Karlsruhe, Germany, pp. 331-338, 2001.

[4] J.P. Barreto, A.Trigo, P. Menezes, J. Dias, A.T.de Almeida, "FBD-The free body diagram method. Kinematic and dynamic modeling of a six leg robot," in: IEEE International Conference on Robotics and Automation, pp. 423- 428, 1998.

[5] P. Gonzalez de Santos, J. Estremera, E. Garcia, "Optimizing leg distribution around the body in walking robots", Proc. of IEEE Int. Conf. on Robotics and Automation, Barcelona, Spain, pp. 3207-3212, 2005.

[6] S. S. Roy, A. K. Singh, and D. K. Pratihar, Analysis of six-legged walking robots, in the Proceedings of the 14thNational Conference on Machine and Mechanisms (NaCoMM-2009), Durgapur, India, 2009,pp. 259-65.

[7] B. Siciliano and O. Khatib, Springer handbook of robotics: Springer, 2008. 
[8] S. S. Roy and D. K. Pratihar, Effects of turning gait parameters on energy consumption and stability of a six-legged walking robot, Robotics and Autonomous Systems, vol. 60, pp. 72-82, 2012.

[9] E. G. P. Gonzalez de Santos, and J. Estremera, Improving walking-robot performances by optimizing leg distribution, Autonomous Robots,vol. 23(4): 247-258, November 2007.

[10] I. Kecskés and P. Odry, Walk Optimization for Hexapod Walking Robot, in the Proceedings of 10th International Symposium of Hungarian Researchers on Computational Intelligence and Informatics, Budapest, Hungary, November, 2009, pp. 12-14.

[11] S. Agarwal, A. Mahapatra, and S. S. Roy, Dynamics and Optimal Feet Force Distributions of a Realistic Four-legged Robot, IAES International Journal of Robotics and Automation (IJRA), vol. 1, pp. 223-234, 2012.

[12] L. H. Ting, R. Blickhan, and R. J. Full, Dynamic and static stability in hexapdal runners. Berkeley: University of California 1994.

[13] J. J. Craig, Introduction to robotics: mechanics and control, Pearson Eduction International, 3rd edition, 2005.

[14] R. N. Jazar, Theory of applied robotics: kinematics, dynamics, and control, Springerverlag Us, 2007. 